Prospect. Univ. (2007) 04(02):75-78

\title{
RENDIMIENTO ACADÉMICO Y AUTOESTIMA EN ESTUDIANTES DE ESPAÑOL Y LITERATURA DE LA FACULTAD DE PEDAGOGÍA Y HUMANIDADES DE LA UNCP*
}

\author{
Cerrón Lozano, Alberto ${ }^{1}$ y Pineda Lozano, Marilú ${ }^{2}$ \\ Facultad de Pedagogía y Humanidades, Departamento Académico de Lingüística, Literatura y Arte. Universidad Nacional \\ del Centro del Perú
}

\section{RESUMEN}

La investigación de tipo básico de nivel descriptivo - correlacional, buscó establecer la relación entre el rendimiento académico y la autoestima de los estudiantes de Español y Literatura de la Facultad de Pedagogía y Humanidades de la Universidad Nacional del Centro del Perú. En su realización se aplicó el método descriptivo con un diseño correlacional. La población estuvo constituida por estudiantes de los cinco semestres de la especialidad de Español y Literatura del ciclo impar 2005, en tanto que la muestra estuvo constituida por 196 alumnos de la especialidad con asistencia regular y que en la encuesta no excedieron el límite de cuatro mentiras. Los resultados indican que entre las variables autoestima y rendimiento académico no existe correlación significativa en el primero, tercero y quinto semestre a excepción del noveno. En el análisis de porcentajes de los niveles de autoestima por sexo, se tiene que tanto varones y mujeres registran una mayor frecuencia en el nivel bajo (59,77\% y 66,97\%, respectivamente), no encontrándose ninguna frecuencia en el nivel alto en ambos sexos.

Palabras clave: rendimiento académico, autoestima, español, literatura.

\section{ACADEMIC PERFORMANCE AND SELF ESTEEM IN STUDENTS OF SPANISH AND LITERATURE SPECIALTY IN THE PEDAGOGY AND HUMAMTIES FACULTY AT UNCP}

\begin{abstract}
The investigation of basic type of descriptive correlational level tried to stablish the relation between the academic performance and self steem in the students of Spanish and Literature speciality of the Pedagogy and humanities faculty at UNCP. In its accomplishment the descriptive method with a correlational design was applied and the Population was conformed by students of the five semesters of spanish and literative speciality of the semesters 2005, where as, the sample was constituited by 196 students of the specially with regular attendance and those in the survey didnit exceed of the limit of four lies. The results indicate that between the selt esteem and Academical Performance variable a significant correlation does not exist in the first, third and fifth semester with the exception of the month. In the analysis of $\%$ of the levels of selfe setee, by sex, we go that both men and women register a high frequency in the lower level ( $59,77 \%$ and $66,97 \%$ respectively) and to frequency was found in high level in both sexes.
\end{abstract}

Key words: academic perfomance, self-esteem., spanish, literature.

\section{INTRODUCCIÓN}

La autoestima y el rendimiento académico son considerados como aspectos importantes en todos los niveles de la estructura educativa, sobre todo, si se tiene en consideración que la autoestima es un tipo particular de actitud hacia uno mismo y que sirve de impulso para el desarrollo personal.
Coopersmith, autor del Inventario de autoestima; fue el psicólogo que más ha trabajado en esta área. Afirma que la autoestima se refiere a la evaluación que una persona hace y comúnmente mantiene con respecto a sí misma. La autoestima expresa una actitud de aprobación 0 desaprobación e indica la medida en que una persona cree ser capaz, importante, exitosa y digna. Otros autores

\footnotetext{
* Este trabajo de investigación fue recibido el 20/01/2006 retornado para su revisión 20/06/2006 y aprobado para su publicación 10/12/2007.

2. malupl7@hotmail.com
} 
señalan que la autoestima es la base y centro del desarrollo humano; es conocimiento, concientización y práctica de todo el potencial de cada individuo.

El rendimiento académico es considerado como el nivel de logro alcanzado en relación a ciertos parámetros establecidos para cada asignatura, después de haber culminado un periodo de estudios. En los centros universitarios, al igual que en colegios secundarios se considera las notas finales como indicador del rendimiento académico de los estudiantes. Para efectos del presente estudio se tomó los promedios ponderados del semestre impar 2005.

La situación de crisis generalizada que atraviesa el Perú, influye en el deterioro de la autoestima de los jóvenes universitarios, que se evidencia con la escasa iniciativa, temor a expresar libremente sus ideas, inseguridad y desconfianza para afrontar situaciones nuevas y también en su descuido personal; aspectos que inciden de manera negativa en su progreso académico, pues la autovaloración que un alumno tiene de sí mismo juega un rol importante en su aprendizaje.

En razón a lo expuesto, se planteó la siguiente interrogante: ¿Qué relación existe entre el rendimiento académico y la autoestima de los estudiantes de la especialidad de Español y Literatura de la Facultad de Pedagogía y Humanidades de la UNCP? En congruencia a ello, el objetivo general del estudio fue determinar el tipo de relación existente entre el rendimiento académico y la autoestima en los estudiantes de la especialidad de Español y Literatura de la Facultad de Pedagogía y Humanidades de la UNCP y, como objetivos específicos se planteó: a) evaluar y caracterizar la autoestima de los alumnos de la muestra de estudio, considerando la variable sexo, b) recoger información de los promedios del rendimiento académico del semestre par del año académico 2005, de los estudiantes de la muestra de estudio, c) relacionar estadísticamente la evaluación de autoestima y el promedio del rendimiento académico de los alumnos de la muestra de estudio, considerando la variable interviniente de sexo.

\section{MATERIAL Y MÉTODOS}

Para la obtención de los datos de la investigación se utilizó la técnica de la observación indirecta y como instrumento, el test autoestima de Coopersmith (1975), que es una prueba diseñada para medir actitudes valorativas hacia sí mismo, en las áreas general, social, hogar y escolar de la experiencia de un sujeto. La investigación se enmarca dentro del tipo de investigación sustantiva, pues está orientada a describir, explicar la realidad; en búsqueda de principios y leyes generales que permita organizar una teoría científica. Se ubica en el nivel descriptivo (Sánchez y Reyes, 1996). El método de investigación utilizado fue el descriptivo con un diseño correlacional.

La población estuvo constituida por estudiantes del I, III, V, VII y IX semestres de la especialidad de Español y Literatura del ciclo impar 2005, Facultad de Pedagogía y Humanidades de la UNCP, que suman un total de 225 alumnos. La muestra estuvo constituida por la totalidad de alumnos de la especialidad con asistencia regular y que en la encuesta no excedieron el límite de cuatro mentiras.

Las variables centrales del estudio fueron el rendimiento académico y la autoestima, y, como variables de control, el sexo de los alumnos.

\section{RESULTADOS}

Tabla 1. Frecuencias de intervalos y niveles de autoestima en la muestra de universitarios de la especialidad de Español y Literatura de la UNCP

\begin{tabular}{cccr}
\hline INTERVALOS & $\begin{array}{r}\text { NIVELES DE } \\
\text { AUTOESTIMA }\end{array}$ & fi & hi \\
\hline 0 a 49 & BAJO & 125 & 63,78 \\
50 a 79 & MEDIO & 71 & 36,22 \\
80 a 100 & ALTO & 0 & 0 \\
& TOTAL & 196 & 100 \\
\hline
\end{tabular}

La tabla $\mathrm{N}^{\circ} 1$ muestra los intervalos y niveles de autoestima en toda la muestra. En ella encontramos 3 niveles que son alto, medio y bajo. La frecuencia más alta se ubica en el nivel bajo con 125 haciendo un 63,78\% y la frecuencia más baja se registra en el nivel medio con 71 de frecuencia y con un porcentaje de 36,22. No se registró frecuencia alguna en el nivel alto.

Tabla 2. Frecuencias de intervalos y niveles de autoestima en la muestra del sexo femenino

\begin{tabular}{cccc}
\hline INTERVALOS & $\begin{array}{r}\text { NIVELES DE } \\
\text { AUTOESTIMA }\end{array}$ & $\mathbf{f i}$ & hi \\
\hline 0 a 49 & BAJO & 73 & 66,97 \\
50 a 79 & MEDIO & 36 & 33,03 \\
80 a 100 & ALTO & 0 & 0 \\
& TOTAL & 109 & 100 \\
\hline
\end{tabular}

En la tabla 2 se observa las frecuencias de intervalos y niveles de autoestima en la muestra del sexo femenino. Donde el nivel bajo registra una frecuencia de 73 con un 
$66,97 \%$ y el nivel medio tiene una frecuencia de 36 con un $33,03 \%$ no encontrándose frecuencia en el nivel alto.

Tabla 3. Frecuencias de intervalos y niveles de autoestima en la muestra del sexo masculino.

\begin{tabular}{cccc}
\hline INTERVALOS & $\begin{array}{c}\text { NIVELES DE } \\
\text { AUTOESTIMA }\end{array}$ & $\mathrm{fi}$ & hi \\
\hline 0 a 49 & BAJO & 52 & 59,77 \\
50 a 79 & MEDIO & 35 & 40,23 \\
80 a 100 & ALTO & 0 & 0 \\
& & 87 & 100 \\
\hline
\end{tabular}

En la tabla $\mathrm{N}^{\circ} 3$ se observa las frecuencias de intervalos y niveles de autoestima en la muestra del sexo masculino. En ella se registra una frecuencia de 52 con un $59,77 \%$ en el nivel bajo, mientras que en el nivel medio se tiene una frecuencia de 35 con un $40,23 \%$ y no encontrándose frecuencia alguna en el nivel alto.

Tabla 4. Correlación entre autoestima y rendimiento académico en universitarios de la especialidad de Español y Literatura de la UNCP

\begin{tabular}{cccccccc}
\hline Autoestima & X & S & Nc & gl & rh & rt & Diagnóstico \\
\hline General & 11,34 & 3,28 & 0,05 & 194 & 0,028 & 0,205 & N.S. * \\
Social & 3,69 & 1,22 & 0,05 & 194 & 0,010 & 0,205 & N.S. ${ }^{*}$ \\
Hogar & 4,14 & 1,12 & 0,05 & 194 & 0,106 & 0,205 & N.S. * \\
Escuela & 3,52 & 1,29 & 0,05 & 194 & 0,091 & 0,205 & N.S. * \\
Total & 45,33 & 9,38 & 0,05 & 194 & 0,038 & 0,205 & N.S. ${ }^{*}$ \\
\hline
\end{tabular}

N.S.*: No Significativo

En la tabla 4, se observa las medias y desviaciones de las áreas general, social, hogar y escuela de la prueba de autoestima, así como los puntajes totales. También se puede apreciar la correlación entre las variables autoestima y rendimiento académico, donde no se halló correlación alguna entre ambas variables, trabajándose con un nivel de confianza de 0,05 y con 194 grados de libertad.

Tabla 5. Correlación entre autoestima y rendimiento académico en universitarias de la especialidad de Español y Literatura

\begin{tabular}{cccccccl}
\hline Autoestima & X & S & nc & Gl & Rh & rt & Diagnóstico \\
\hline General & 11,21 & 3,17 & 0,05 & 107 & 0,201 & 0,205 & N.S.* \\
Social & 3,72 & 1,20 & 0,05 & 107 & 0,083 & 0,205 & N.S. $^{*}$ \\
Hogar & 4,21 & 1,15 & 0,05 & 107 & 0,108 & 0,205 & N.S. $^{*}$ \\
Escuela & 3,50 & 1,34 & 0,05 & 107 & 0,090 & 0,205 & N.S. $^{*}$ \\
Total & 45,45 & 8,75 & 0,05 & 107 & 0,169 & 0,205 & N.S. $^{*}$ \\
\hline N.S.*: No Significativo & & & & &
\end{tabular}

La tabla 5, nos muestra los resultados de la correlación de las variables autoestima y rendimiento académico en las estudiantes universitarias. Notándose que en ninguna área de la prueba de autoestima se registra correlación estadística alguna y mucho menos en los puntajes totales.

Tabla 6. Correlación entre autoestima y rendimiento académico en universitarios de la especialidad de Español y Literatura.

\begin{tabular}{|c|c|c|c|c|c|c|c|}
\hline Autoestima & $a x$ & $\mathrm{~S}$ & Nc & GI & rh & rt & Diagnóstico \\
\hline General & 11,51 & 3,42 & 0,05 & 85 & 0,135 & 0,217 & N.S. \\
\hline Social & 3,67 & 1,25 & 0,05 & 85 & $-0,062$ & 0,217 & N.S. \\
\hline Hogar & 4,05 & 1,08 & 0,05 & 85 & - 0,181 & 0,217 & N.S. \\
\hline Escuela & 3,53 & 1,23 & 0,05 & 85 & $-0,103$ & 0,217 & N.S. \\
\hline Total & 45,17 & 10,16 & 0,05 & 85 & 0,042 & 0,217 & N.S. \\
\hline
\end{tabular}

Ns: No significativo

La tabla 6, nos muestra los resultados de la correlación de las variables autoestima y rendimiento académico en los estudiantes universitarios. En ninguna área de la prueba de autoestima se registra correlación estadística alguna y mucho menos en los puntajes totales.

Tabla 7. Correlación entre autoestima y rendimiento académico por semestres.

\begin{tabular}{lccccccc}
\hline Autoestima & X & S & nc & gl & Rh & rt & Diagnostico \\
\hline PRIMERO & 48,79 & 9,18 & 0,05 & 41 & 0,174 & 0,304 & Ns \\
TERCERO & 43,21 & 7,42 & 0,05 & 54 & 0,207 & 0,273 & Ns \\
QUINTO & 44,59 & 10,00 & 0,05 & 39 & 0,069 & 0,325 & $\mathrm{Ns}$ \\
SÉPTIMO & 46,81 & 12,12 & 0,05 & 30 & 0,132 & 0,349 & $\mathrm{Ns}$ \\
NOVENO & 43,33 & 6,84 & 0,05 & 22 & 0,442 & 0,423 & $\mathrm{~S}$ \\
\hline
\end{tabular}

Ns: No significativo S: Significativo

La tabla 7, nos muestra los resultados de la correlación de las variables autoestima y rendimiento académico en los estudiantes universitarios del primero, tercero, quinto, séptimo y noveno semestre. Encontrándose sólo correlación de ambas variables en el noveno semestre y no registrándose correlación alguna en los demás semestres académicos.

\section{DISCUSIÓN}

El psicólogo Coopersmith, S., propone en términos generales que siendo la autoestima una actitud hacia uno mismo, tiene los tres componentes que caracterizan toda actitud: cognitivo, afectivo y reactivo. El autor afirma que los individuos con elevada autoestima se acercan a personas que realizan tareas con considerables expectativas de éxito, 
aceptan sus opiniones y manifiestan confianza en sus relaciones, mostrando mayor independencia social y creatividad, lo que los conduce a comportamientos más asertivos, agregando que el nivel de autoestima de los niños y jóvenes es esencial para su desarrollo educativo.

Tanto las investigaciones realizadas como la experiencia de educadores, profesores, asesores y psicólogos indican que la mayoría de estudiantes con problemas y dificultades de aprendizaje proceden de hogares conflictivos y evidencian problemas de autoestima. Ello significa que entre autoestima y rendimiento académico existe una relación directa. En contraste a ello en los resultados de la presente investigación, en la cual se ha establecido la relación entre ambas variables, no se ha encontrado relación estadísticamente significativa entre ellas, lo que estaría entrando en contradicción en la teoría sobre el tema.

En la tabla referida a frecuencias de intervalos y niveles de autoestima en la muestra de universitarios de la especialidad de Español y Literatura de la UNCP se consideró tres niveles de autoestima con sus correspondientes intervalos: un nivel bajo, con un intervalo de 0 a 49: otro medio, de 50 a 79 y finalmente uno alto, con un intervalo de 80 a 100 puntos. En ella podemos observar el siguiente panorama. El porcentaje más alto se halla en el nivel bajo con $63,78 \%$, en contraste con un $0 \%$ de frecuencia en el nivel de autoestima alto.

En primer término los resultados hallados en los tres niveles de autoestima, nos dan un indicador muy importante: que el nivel de autoestima de los estudiantes universitarios de la especialidad de Español y Literatura de la UNCP es alarmantemente bajo, lo que indubitablemente tiene que influir negativamente en su rendimiento académico. En un segundo término hallamos un porcentaje de $36,22 \%$ de frecuencia en el nivel medio, constituyéndose en un cincuenta por ciento de la frecuencia que presenta el nivel bajo.

\section{CONCLUSIONES}

- De acuerdo a los resultados obtenidos al término de nuestra investigación, la autoestima de los estudiantes de la especialidad de Español y Literatura de la Facultad de Pedagogía y Humanidades de la Universidad Nacional del Centro del Perú, correspondiente al Año Académico 2005-I, un $63,78 \%$ presenta una autoestima baja, un 36 , $22 \%$ media y un $0 \%$ una autoestima alta.

- Los resultados desde la perspectiva de la variable control género arroja los siguientes resultados: en la muestra de sexo femenino que suma un total de 109 alumnas, el $66.97 \%$ se ubica en un nivel de autoestima baja en contraste con un $33.03 \%$ de alumnas que hace un total de 36 , que presentan una autoestima media.

- En lo que respecta a los resultados en la muestra de sexo masculino nos brinda el siguiente panorama: con un nivel de autoestima baja se encuentra un total de 52 alumnos haciendo un porcentaje de $59.77 \%$ en tanto que 35 estudiantes que hace un porcentaje de 40,23 , se encuentra en un nivel de autoestima media. Resulta bastante llamativo que ningún alumno ni alumna haya alcanzado un nivel de autoestima alta.

- En lo que respecta a la relación entre nivel de autoestimay rendimiento académico no existe correlación alguna entre ambas variables, trabajándose con un nivel de confianza de 0,05 y con 194 grados de libertad. Este resultado desestima la hipótesis planteada de que existe una correlación positiva entre el rendimiento académico y la autoestima, en el presente caso, de los alumnos de la especialidad de Español y Literatura de la Facultad de Pedagogía y Humanidades de la UNCP. Es menester aclarar que, a excepción del noveno semestre, en ninguna de las áreas de la prueba de autoestima se registra correlación estadística alguna y mucho menos en los puntajes totales, tal como se puede apreciar en las Tablas $5 \mathrm{y} 7$.

- Todos estos resultados nos dan un indicador muy importante: que el nivel de autoestima de los estudiantes universitarios de la especialidad de Español y Literatura de la UNCP es alarmantemente bajo, lo que indubitablemente tiene que influir negativamente en su rendimiento académico. En un segundo término hallamos un porcentaje de $36,22 \%$ de frecuencia en el nivel medio, constituyéndose en un cincuenta por ciento de la frecuencia que presenta el nivel bajo.

\section{LITERATURA CITADA}

Cárdenas, B. 1989. Autoestima en niños urbano-marginales y su relación con el nivel de soporte social brindado por padres y pares. Tesis, USMP, Lima.

Casimiro, C. y Mucha, E. 1999. Modulo de habilidades sociales para el desarrollo de la autoestima de los alumnos del segundo grado de la E.E. N ${ }^{a}$ 31555- Jauja. Tesis, UNCP, Huancayo.

Coopersmith, S. 1990. SEl: Self - Esteem-Inventories. Consulting Psychologists Press. Palo Alto, California.

Llerena, A. 1995. Niveles de la autoestima y normalización del inventario de autoestima de Stanley Coopersmith en adolescentes de centros educativos de gestión estatal y no estatal de Lima Metropolitana. Tesis, URP, Lima-Perú.

Loli, A. y López, E. 2002. La autoestima y los valores organizacionales en estudiantes universitarios y no estudiantes universitarios de Lima. Revista de Investigación en Psicología. IIPSI-UNMSM. Instituto de Investigaciones Psicológicas Vol.5, N 1 . Lima -Perú, p. 141-155

Yarleque, L. y otros. 2002. Psicología evolutiva y pedagógica. UNCP. Facultad de Pedagogía y Humanidades. 2da. Edición, Huancayo-Perú. 